\title{
Le point de vue d'un élu sur les SDAGE
}

\author{
par Paul-Louis Tenaillon
}

Député des Yvelines, Président d'Honneur du Conseil général

Le 18 septembre 1990, je déposais sur le bureau de l'Assemblée nationale une proposition de loi sur l'eau ( $\mathrm{n}^{\circ} 1620$ ), assortie du commentaire suivant : "Du fait de la spécificité des problèmes que pose l'eau et des différentes solutions qui peuvent leur être apportées, il nous parait essentiel qu'entre toutes les parties concernées par une même ressource, un cours d'eau et sa nappe par. exemple, puisse être créée une autorité organisatrice des eaux pour préserver la qualité de la ressource et harmoniser l'utilisation entre les différents acteurs... »

Quelques mois plus tard, le texte du gouvernement débattu à l'Assemblée, et qui débouchera sur la loi sur l'eau du 3 janvier 1992, retiendra cette idée fondamentale, sous les appellations de SAGE et de SDAGE, dans les conditions que vous a rappelées, ce matin, Jean-Loïc Nicolazo.

Autorité organisatrice ou SDAGE/SAGE, quid du vocabulaire, chacun - et en premier lieu les élus - était conscient de la nécessité, de l'urgence d'organiser une gestion locale, à l'échelle du bassin et des sous-bassins, associant l'ensemble des partenaires intéressés, pour définir des priorités et une règle du jeu commune. Ceci, bien entendu, dans le cadre de perspectives à long terme, définies en commun.

L'idée maîtresse est ici la concertation.

Une concertation propre à résoudre les conflits entre des usages, tous aussi légitimes les uns que les autres. Celle-ci doit devenir coopération; elle prend toute son ampleur dans le cadre du SAGE, d'initiative locale, par l'intermédiaire de la Commission Locale de l'Eau. Encore faut-il que cette démarche reste cohérente avec les grandes orientations définies à l'échelle du bassin et fixées par le SDAGE.

Dans l'élaboration d'un SDAGE, l'Etat chargé dẹ veiller aux grands équilibres, et à qui il appartient de faire respecter les dispositions législatives nationales ou communautaires, dispose d'un large pouvoir. Il reste que ces orientations encadrant la politique de I'eau doivent s'avérer compatibles avec celles des autres partenaires: collectivités territoriales, représentant des usagers. Et c'est la recherche du consensus, aux différents stades d'élaboration et sur les différents aspects techniques, juridiques, économiques, qui prévaut généralement. Un consensus qui vise à défnir les grandes orientations, et qui ne comporte pas d'engagement préalable sur les investissements.

Les SDAGE sont en cours d'élaboration dans chacun des six grands bassins hydrographiques, avec une prévision d'achèvement pour 1996, conformément aux dispositions de la loi sur l'eau qui prévoyait un délai maximal de 5 ans. Des commissions travaillant sous l'égide des Comités de bassin, avec des approches croisées géographiques et thématiques, ont permis l'établissement d'un premier document d'orientation.

Les commissions travaillent généralement sur le territoire d'un sous-bassin, avec toutefois une particularité pour Seine-Normandie, où le caractère spécifique de la Région a conduit à la création d'un intergroupe Ile-de-France, que j'ai l'honneur de présider.

Dans cette région, dont l'impact économique et démographique pèse sur l'ensemble du bassin, une bonne part des écoulements d'eau n'empruntent pas les rivières, mais les canalisations et les égouts; par ailleurs, il était important d'avoir une réflexion à l'échelle régionale comme pour le nouveau schéma directeur, accouché dans la douleur en 1994, et dont les implications sur les problèmes d'eau, ressource, assainissement, ruissellement, sont nombreuses.

Après un examen thématique approfondi, les travaux de I'intergroupe ont débouché sur un certain nombre de préconisations concernant la ressource en eau, les eaux usées, les inondations, la sauvegarde du patrimoine naturel, etc.

Ceux qui ont ainsi participé étroitement à l'élaboration d'un SDAGE, ont pu en mesurer tout l'intérêt, mais aussi les difficultés: approches techniques différentes, conflits d'intérêts, conciliation des intérêts particuliers avec l'in- 
térêt général, tentative par l'Etat, dans certains cas, de faire évoluer l'outil de planification vers le domaine réglementaire,...

Mais la principale difficulté réside dans l'établissement d'un document suffisamment précis pour fixer les grandes orientations de la politique de l'eau dans le bassin, mais qui reste général pour ne pas constituer un guide de programmation, susceptible de réduire la capacité d'action des acteurs locaux, à qui revient l'initiative et la responsabilité des réalisations, dans le cadre des SAGE.
Ce sont les difficultés que soulignait, tout à l'heure, le Directeur de l'Agence de l'Eau Loire-Bretagne. Il ne faut ni les exagérer, ni les sous-estimer. Jean-Luc Laurent disait ainsi au Congrès des Maires de France en 1994: «Les outils sont en place, il faut les laisser vivre. " Personnellement, je fais confiance aux différentes parties prenantes pour débattre ensemble, avec «ces outils», de l'avenir de l'eau dans chacun des bassins versants.

\section{Procédure de mise en place des SDAGE et SAGE}

Les SDAGE doivent être élaborés par les Comités de Bassin compétents, au plus tard le 3 janvier 1997, et fixent, pour chaque bassin, les orientations fondamentales d'une gestion équilibrée de la ressource.

Les SAGE, à un niveau plus local, sont élaborés, sur la base d'un périmètre géographique bien défini, par une structure administrative nouvelle: la Commission Locale de l'Eau (C.L.E.).

Elle se compose de trois collèges différents :

- pour moitié, des représentants des collectivités territoriales et établissements publics locaux,

- pour un quart, des représentants des usagers, propriétaires riverains, organisations professionnelles et associations concernées,

— pour un quart, des représentants de l'Etat et de ses établissements publics.

\section{Objectif général des SDAGE et des SAGE}

Rechercher un consensus durable entre, d'une part la restauration, la protection, la mise en valeur des milieux aquatiques (écosystèmes, qualité générale de l'eau) et d'autre part, le développement et la satisfaction des usages liés à la ressource.

\section{Orientations à respecter}

1. Reconnaissance, en tant que telle, des écosystèmes aquatiques et de leur fonction intrinsèque de régulation (débits, maintien de la qualité de l'eau).

2. Adaptation des usages aux potentialités réelles des milieux (prélèvements et rejets).

3. Priorité donnée à une meilleure gestion des ouvrages et espaces existants, avant la réalisation de nouveaux aménagements. 


\section{Présentation}

\section{DES \\ NAPPES PROFONDES}

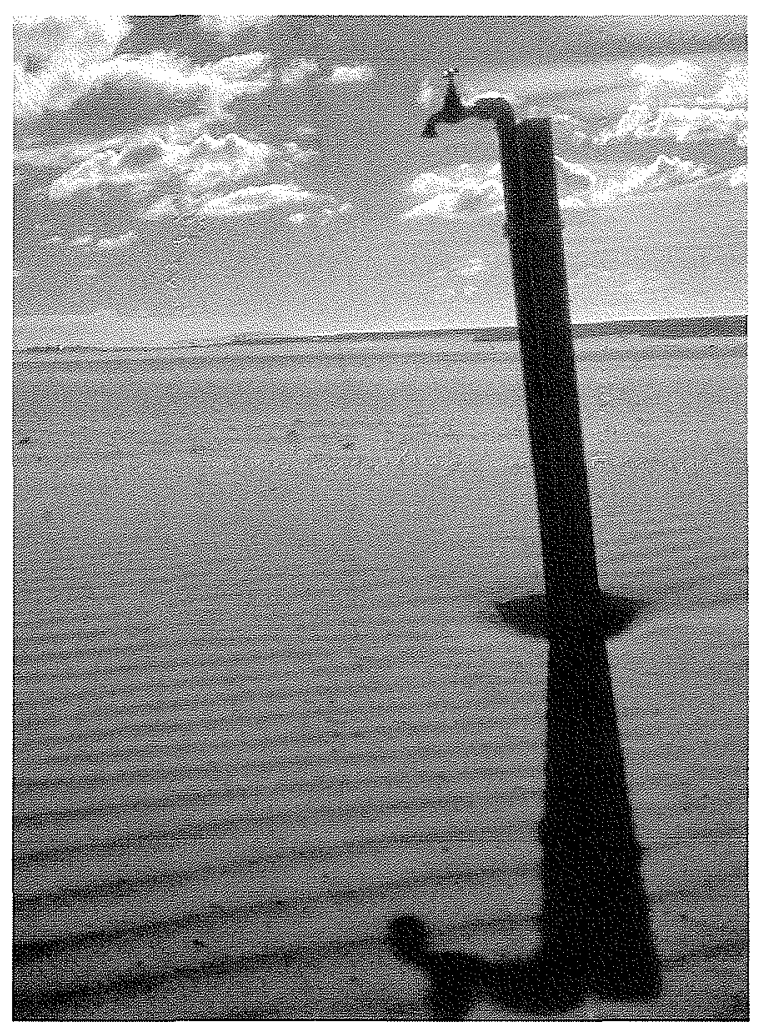

Les nappes profondes constituent des réserves de plus en plus sollicitées par les pouvoirs publics. Il s'agit de systèmes aquifères multicouches, généralement de dimension régionale, caractérisés par des stocks importants et des flux faibles, avec un temps de séjour de l'eau relativement long. S'il s'agit de systèmes fïssurés karstiques, ces nappes peuvent affleurer localement, ce qui permet leur alimentation.

Les eaux des nappes profondes sont naturellement plus minéralisées et souvent de température élevée. La vulnérabilité à la pollution est réduite dans les zones profondes bien qu'il existe des pollutions naturelles liées à la dolomitisation, aux lacunes, variations de faciès etc.

En revanche, l'exploitation intensive des aquifères peut provoquer des échanges couche à couche et altérer la qualité de la ressource. Des variations de la piézométrie, des tassements de sols peuvent être compensés par la réalimentation artificielle des nappes.

Les prélèvements ou les réinjections d'eaux en équilibre avec leur milieu entraînent des phénomènes de précipitation, de dissolution ou de corrosion.

L'utilisation des nappes pour le stockage des gaz, pour l'exploitation pétrolière ou minière, ou encore pour leurs eaux thermales, constitue aussi un outil privilégié de connaissance de leur fonctionnement. 